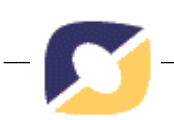

\title{
C:-)LMEIAS: Uma Estratégia Didático-Pedagógica que Interliga as Aprendizagens Móvel, Colaborativa e Significativa
}

\author{
Silvana Letícia Pires Iahnke, PPGEC/FURG, CINAT-Matemática/IFSUL, email: \\ sille@pelotas.ifsul.edu.br
}

Silvia Silva da Costa Botelho, PPGEC/FURG, email: silviacb@furg.br

André Luis Andrejew Ferreira, PPGECM/DME/UFPel, email: andreferreira@ufpel.edu.br

Resumo: Vive-se na atualidade numa sociedade reconhecida como a Sociedade da Informação, em um mundo em que as tecnologias digitais e móveis passam cada vez mais a fazer parte do cotidiano das pessoas. Diante desse novo cenário, com a finalidade de promover uma educação mais coerente e contextualizada com essa realidade, o presente artigo procura apresentar uma breve revisão sobre o paradigma computacional atual, a aprendizagem móvel e as redes sociais, apresentando a proposta de uma estratégia didático-pedagógica, denominada como C):LMEIAS (Estratégia DidáticoPedagógica que Interliga as Aprendizagens Móvel, Colaborativa e Significativa), a qual tem por finalidade facilitar a aprendizagem significativa através da colaboração nas redes sociais num contexto de mobilidade.

Palavras-Chave: Aprendizagem, Aprendizagem Móvel, Aprendizagem Significativa, Colaboração.

Abstract: We live today in a society known as the Information Society, in a world where digital and mobile technologies make increasingly part of everyday life. In this new context, in order to promote a more coherent education with this reality, this article presents a brief review of the current computing paradigm, mobile learning and social networking, presenting an proposed strategy didactic-pedagogic called C;:LMEIAS, which aims to facilitate meaningful learning through collaboration in social networks in the context of mobility.

Keywords: Learning, Mobile Learning, Meaningful Learning, Collaboration.

\section{C:-)LMEIAS: An Didactic-Pedagogical Strategy that Interconnects the Learnings Mobile, Collaborative and Meaningful}

\section{Introdução}

Na atualidade cada vez mais as tecnologias digitais e móveis tornam-se parte de nosso cotidiano, a tal ponto que hoje a "janela é a tela", em que a interação com os outros indivíduos e instituições de todo o mundo é realizada através de nossos computadores, de nossos celulares (Kenski, 2010).

Nesse contexto, as Tecnologias da Informação e Comunicação (TICs) afetam "praticamente todos os âmbitos de atividades das pessoas, desde as formas práticas de organização social até o modo de compreender o mundo, de organizar essa compreensão e de transmiti-las a outras pessoas” (Coll, Monereo, 2010, p.17).

São várias as mudanças tecnológicas que nosso meio vem sofrendo, refletindo também na forma como a nossa sociedade se organiza. Essa vem sendo reconhecida, de 
acordo com Coll e Monereo (2010), como a Sociedade da Informação (SI), a qual representa um novo estágio de desenvolvimento das sociedades humanas, caracterizada pelo acesso facilitado à informação, ao seu compartilhamento, de maneira praticamente instantânea, a partir de qualquer local e na forma preferida.

Na SI destaca-se que tanto a produção como a aquisição do conhecimento são processos distintos do de épocas anteriores. Para o sucesso dos processos educativos nessa sociedade, torna-se necessário que o papel docente leve em consideração o perfil dos discentes desse novo século, uma vez que os mesmos são "nativos digitais”, isto é, alunos que são focados em conexão e interação social, com preferência por atividades baseadas em grupo e ocasiões sociais, que desenvolvem suas vidas em um contexto no qual o ciberespaço torna-se parte constituinte de seu dia a dia (Cobcroft et al., 2006).

Nesse novo milênio, para que o processo de ensino e de aprendizagem seja mais coerente com a realidade atual e o perfil desses aprendizes, mudanças na forma como se concebe e se faz a educação tornam-se cada vez mais necessárias. Além disso, salientase que a mobilidade dos serviços computacionais, bem como a diversidade de recursos que esses possuem, viabiliza que também a aprendizagem possa ocorrer em qualquer lugar, a todo instante, através de diferentes dispositivos, ou seja, que os processos de aprendizagem móvel ou mobile-learning ${ }^{1}$ sejam estabelecidos.

A aprendizagem com mobilidade vem sendo considerada como o futuro da aprendizagem ou como parte integrante de qualquer outra forma de processo educativo no futuro (Trinfonova, 2003; Lam, Yau, Cheung, 2010). Segundo Wu et al. (2012), essa já é uma das principais tendências de uso das tecnologias em aplicações educacionais.

Nesse contexto tecnológico e móvel, os sites de redes sociais atraem milhares de milhões de usuários, fazendo parte de suas rotinas diárias (Yu, Zhu, 2011). As redes sociais transformam a realidade de nossos educandos, podendo ser definidas como "ferramentas virtuais que têm como objetivo facilitar a comunicação entre pessoas ou grupo de pessoas” (Espada, 2012, p. 51).

A educação nesse novo cenário caminha

para uma flexibilização forte de cursos, tempos, espaços, gerenciamento, interação, metodologias, tecnologias, avaliação. Isso obriga a experimentar, pessoal e institucionalmente, modelos de cursos, de aulas, de técnicas, de pesquisa, de comunicação.

Todas as universidades e organizações educacionais, em todos os níveis, precisam experimentar formas de integrar o presencial e o virtual, garantindo a aprendizagem significativa (Moran, 2002, p. 273, grifo nosso).

Assim, apropriando-se do fato de que as redes sociais estão cada vez mais presentes nas relações e no cotidiano dos indivíduos, e que, segundo Almeida et al. (2000), o meio que por excelência vem favorecendo, em qualquer local do mundo e em qualquer hora, a colaboração entre as pessoas é a Internet e os seus serviços, é possível levantar a questão da aprendizagem colaborativa através das redes sociais a partir de dispositivos móveis com a finalidade de promover à aprendizagem significativa.

Dessa forma, a fim de contribuir nesse processo de mudança, procura-se apresentar através do presente artigo, a proposta de uma estratégia didático-pedagógica, a C):LMEIAS, que tem por finalidade facilitar a aprendizagem significativa através da aprendizagem colaborativa num contexto de aprendizagem móvel. Dessa forma a próxima seção investigará as influências desencadeadas em nosso meio pela mudança dos paradigmas computacionais, seguida por questões relacionadas à aprendizagem móvel e as redes sociais, para então ser apresentada essa estratégia. Por último são indicadas as considerações finais e as referências bibliográficas.

\section{Paradigmas Computacionais x Educação}


No contexto atual, é possível identificar a transição dos paradigmas computacionais, da era dos computadores pessoais ${ }^{2}$ (PC) para a era da computação ubíqua $^{3}$ (UC), sendo esse período marcado pelo rápido desenvolvimento da computação móvel, cujas propriedades essenciais são a comunicação, a mobilidade, a portabilidade e a adaptação. O fato dos computadores serem portáteis e com a capacidade de usar as redes sem fio vem revolucionando a forma como os computadores são empregados (Forman, Zahorjan, 1994; Satyanarayanan, 1996).

Segundo a IDC ${ }^{4}$, as atividades mais populares dos usuários da Internet, em 2010, consistiam em consultar sites de busca, ler notícias, realizar downloads de multimídia, usar o email e programas de mensagens instantâneas, sendo esperado que até 2014, as atividades que mais cresçam nesse setor seja o emprego das redes sociais e dos blogs, os quais, passarão em breve a ser acessados principalmente por meio de uma grande quantidade (e variedade) de dispositivos móveis (Mascolo, 2010).

Esses são produzidos com objetivo de serem cada vez mais rápidos em relação a sua conectividade, sendo a cobertura das redes ampliada e a velocidade de transferência cada vez maior, estimando-se que no futuro, com a tecnologia $4 \mathrm{G}$, que a velocidade possa ser superior a 20 Mbps (Hart, Hannan, 2004). A geração 4G das redes está relacionada, segundo $\mathrm{ITU}^{5}$, as tecnologias WiMAX ${ }^{6}$ e o $\mathrm{LTE}^{7}$ e permitirá a conexão à Internet em movimento, com alta velocidade de acesso móvel, sem fio e na transmissão de dados (Srivastava, Srivastava, 2012).

As redes 4G possibilitarão ainda que a conectividade torne-se onipresente em nosso meio, tornando a Internet potencialmente disponível para todos, em todos os lugares, sendo as barreiras removidas e a colaboração melhorada. Elas representam uma evolução na forma como a Internet será empregada, trazendo muitas oportunidades para as mais diversas áreas. Na educação, cada vez mais os processos de m-learning poderão ser estabelecidos, sem barreiras de tempo ou lugar (IBIDEM).

A aprendizagem móvel é, majoritariamente, definida como a aprendizagem que ocorre vinculada aos dispositivos móveis (Laouris, 2005; Winters, 2006). É uma área que vem se desenvolvendo rapidamente nos últimos anos, tanto em termos de tecnologias associadas à mesma, como em relação à pedagogia (Traxler, 2007), tornando-se um componente viável e rico de possibilidades de apoio à educação. Para melhor compreendê-la dedica-se a próxima seção para a sua revisão mais detalhada, juntamente com as redes sociais, uma vez que as mesmas estão cada vez mais onipresentes no cotidiano das pessoas.

\section{Aprendizagem Móvel e Redes Sociais}

A aprendizagem móvel começou a ser estudada a pouco mais de uma década, tendo seu interesse na área da educação sido renovado nos últimos anos, com o avanço e desenvolvimento das TICs, principalmente daquelas tecnologias associadas ao paradigma da computação móvel, tal como o smartphone (Asabere, 2012).

Essa é um novo paradigma educacional que possibilita uma aprendizagem mais personalizada, integrada, situada, contextual, espontânea, cooperativa e centrada no indivíduo, favorecendo a ocorrência efetiva da aprendizagem colaborativa/social quando associada a uma metodologia de ensino e de aprendizagem adequada.

Segundo Asabere (2012) a aprendizagem colaborativa/social é uma metodologia em que os alunos trabalham juntos em pequenos grupos para um objetivo comum. Nessa o professor atua como um orientador ou facilitador do processo de aprendizagem, sendo a realização bem sucedida do objetivo comum, compartilhada entre todos os membros do grupo. Os alunos devem tomar a iniciativa, sendo responsáveis pela sua 
aprendizagem. Os aprendizes trabalham em conjunto em uma tarefa, trocam experiências e opiniões, discutem e negociam estratégias, ações e resultados. Ajudam a explicar, ensinar, compreender, analisar e influenciam uns aos outros mutuamente no processo. Através do desenvolvimento de uma comunidade de aprendizagem eles combinam as habilidades de cada um para alcançar o objetivo comum.

Várias são também as vantagens pedagógicas citadas na literatura para o uso da aprendizagem móvel na educação, entre as quais destacam-se: a ubiquidade, a portabilidade, a flexibilidade, a conectividade, o baixo custo, a facilidade de uso, a familiaridade, a mobilidade, o aumento de produtividade e os aspectos cognitivos (vídeo, áudio, imagens, texto); possibilitando atividades mais criativas, críticas e comunicativas, que integram o virtual com o presencial, que favorecem a autoaprendizagem, a autoestima e a autoconfiança.

Com o rápido desenvolvimento das TICs e pelas mudanças desencadeadas em nossa sociedade, as abordagens didático-pedagógicas dos espaços físicos e virtuais cada vez mais requerem uma reavaliação por parte da comunidade acadêmica, uma vez que os aprendizes da atualidade são nativos digitais. Nesse contexto, salienta-se que a ampla disponibilidade de tecnologias móveis e sem fio são fundamentais, porém se sozinhas, são insuficientes para ambientes de aprendizagem eficazes. Para o sucesso de projetos envolvendo a aprendizagem móvel, mudanças tanto no comportamento dos alunos como das Instituições de Ensino serão necessárias. Métodos para a aprendizagem online precisam ser revistos como novos contextos de aprendizagem e não apenas como ferramentas (Cobcroft et al., 2006).

Enfim torna-se necessário o desenvolvimento de novas estratégias para o ensino e a aprendizagem que possibilitem um espaço de conversa compartilhada, que utilizem as tecnologias móveis, que promovam a colaboração entre os estudantes, que reconheçam que a aprendizagem colaborativa torna-se efetiva quando as pessoas podem conversar umas com as outras, compartilhando suas descrições do mundo.

Nesse cenário, de acordo com Al-Husain e Hamo (2013), há necessidade de pensar-se a aprendizagem móvel como autodirigida e colaborativa, onde a Web é um espaço de informações, no qual as pessoas podem se comunicar de forma especial, compartilhando o seu conhecimento, sendo essencial reconhecer que as redes e as comunidades sociais estão crescendo rapidamente, conectando pessoas com interesses semelhantes, representando uma revolução social, não apenas uma revolução tecnológica, sendo os serviços associados às mesmas adotados por diversos usuários em todo o mundo e a tendência atual estar em estreita comunicação com as outras pessoas através de Sites de Redes Social na Internet (RSI), tal como o Facebook, Twitter, MySpace, entre outros.

Esses sites estão sendo adotados rapidamente e, de acordo com Petrovic et al. (2012), a maioria de seus usuários são estudantes. Estudos demostram que as ferramentas dessas redes são capazes de apoiar as atividades de ensino, tornando-as mais interativas e colaborativas, possibilitando uma participação ativa, no compartilhamento dos recursos, da informação e do pensamento crítico, destacando-se o Facebook para o uso educacional, já que o mesmo contém as potencialidades necessárias para ser usado como um ambiente de aprendizagem e de ensino, sendo adotado como uma extensão da sala de aula tradicional.

O Facebook é um RSI de comunicação síncrona e assíncrona, que possibilita o acompanhamento do discente, oferecendo ao mesmo oportunidades de definir seus próprios caminhos de aprendizagem, demandando uma participação colaborativa. Esse ainda apresenta uma variedade de recursos - fóruns de discussão, chat, mural, fotos, vídeos, agenda, mensagens, notícias, entre outros - que podem potencializar o processo 
5

de ensino e de aprendizagem quando associados a uma metodologia apropriada. (Oliveira, Pimental, Mercado, 2011).

Nesse cenário salienta-se que o principal desafio para os educadores encontra-se na mudança conceitual que precisa ser realizada para promover a colaboração no lugar da competição nas salas de aula. Os aprendentes precisarão criar significados nesse contexto, o que requer um equilíbrio entre a autonomia e a capacidade de aprender de forma independente e em colaboração, com base em um processo de interpretação e (re)construção dos saberes, de uma variedade de experiências na comunidade de aprendizagem, num ambiente onde o que é significativo para o sujeito é criado através da interação social (Frías, Montaño, 2012).

É ainda recomendável, para o sucesso do processo de ensino e de aprendizagem nesses contextos,

criar metodologias de abordagem que estimule o interesse dos alunos na participação das atividades propostas pelo professor e neste processo o professor deve ser sempre a figura central desta relação. [...] não basta que os alunos simplesmente acessem as informações, para que estas tecnologias sejam significativas, eles precisam ter a habilidade de utilizar, relacionar, sintetizar, analisar e avaliar. $O$ professor deve estar o tempo todo como mediador, desafiando os alunos a irem além de respostas simples, desafiando ideias e conclusões (Paixão et al., 2012, p.2431, grifo nosso).

\section{A Estratégia Didático-Pedagógica - C:-)LMEIAS}

Reconhecendo a familiaridade que os estudantes da atualidade têm com o Facebook, que através do mesmo as interações sociais podem ocorrer não apenas em um grupo fechado, mas entre todos que estão envolvidos nessa rede e que esse possui uma diversidade de recursos, podendo ainda ser acessado por uma variedade de dispositivos móveis, a estratégia didático-pedagógica que será proposta adotará esse site como um ambiente pessoal de aprendizagem ${ }^{8}$, com a finalidade de aproximar efetivamente os processos educativos com a realidade desses aprendizes.

Através de um PLE, segundo Barcelos, Passerino e Behar (2011), a aprendizagem poderá ocorrer em diferentes contextos e situações, ampliando o acesso à educação, uma vez que poderá relacionar a aprendizagem da vida e a aprendizagem da escola ou da universidade. Nesse ambiente o aprendente empregará um conjunto único de ferramentas, personalizadas de acordo com as suas preferências e necessidades.

Para o desenvolvimento de uma estratégia nesse contexto torna-se necessário identificar que o processo de ensino e de aprendizagem nesse cenário tecnológico e móvel será um processo mediado pela interatividade social, pela sincronidade e pela negociabilidade, onde os parceiros não impõem seus pontos de vistas, mas os negociam, os defendem e os justificam através do diálogo (Dillenbourg, 1999).

Além disso, a partir da aprendizagem social/colaborativa os aprendizes deverão reconsiderar seus conceitos através da discussão com os integrantes do grupo (colegas e professores), fazendo "coisas" e refletindo sobre as mesmas, pois nesse tipo de aprendizagem espera-se que "os alunos se comprometam com a sua aprendizagem e aprendam a aprender junto com os outros, que seja também sua responsabilidade a aprendizagem dos colegas e onde o êxito do grupo depende de cada um e de todos" (Alcântara et al., 2004, p. 4).

Através de práticas que envolvam a colaboração dos estudantes, almeja-se que a aprendizagem dos mesmos seja significativa, a qual de acordo com Santos (2009, p. 62) é aquela que "ocorre a partir do surgimento de um sentido pessoal por parte de quem aprende, o que desencadeia uma atitude proativa que tenta desvendar o novo e reconstruir conceitos que ampliam cada vez mais a habilidade de aprender”. 
Portanto, com a finalidade de promover a aprendizagem significativa através de um processo colaborativo nas redes sociais em contextos de mobilidade, a estratégia que está sendo desenvolvida relacionará as três modalidades de aprendizagem: móvel, colaborativa e significativa, sendo nomeada como C@-LMEIAS (Estratégia DidáticoPedagógica que Interliga as Aprendizagens Móvel, Colaborativa e Significativa).

Dessa maneira, a COLMEIAS procurará (re)construir o conhecimento de forma significativa, a partir de um processo colaborativo que empregue os recursos do Facebook associados a sala de aula presencial, sendo seu objetivo expresso na Figura 1.

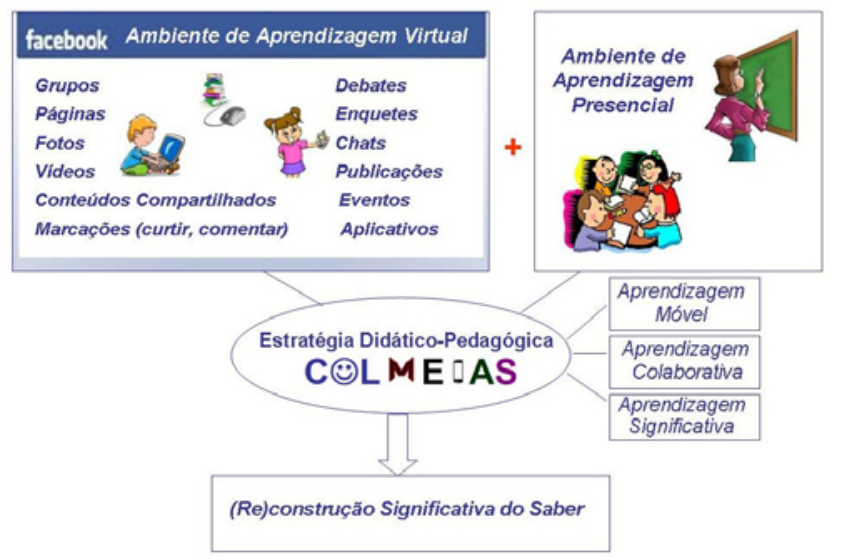

Figura 1 - Objetivo da COLMEIAS

Considerando os princípios programáticos e as estratégias facilitadoras propostas na teoria de Ausubel para a aprendizagem significativa, apresentadas por Moreira (1999), e identificando que a C@-)LMEIAS será aplicada tanto no ambiente virtual como presencial, que o seu movimento não será linear, uma vez que os conhecimentos poderão ser (re)construídos em qualquer lugar, através de diferentes dispositivos e a todo instante, compartilhados no site de rede social de forma síncrona e assíncrona, que o aluno será incentivado a aprender pela descoberta, dos conceitos mais gerais para os mais específicos, resgata-se da proposta de ensino de Jerome Bruner ${ }^{9}$ o currículo em espiral. Assim, a dinâmica dessa estratégia é representada pela Figura 2.

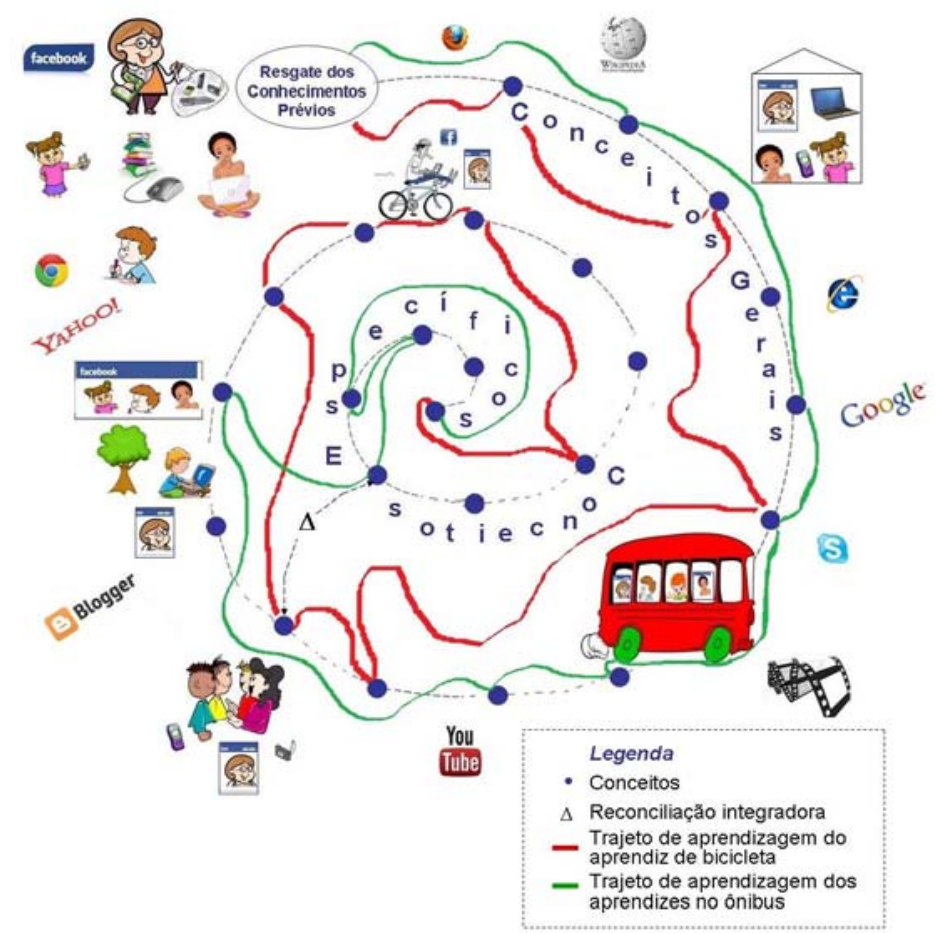

Figura 2 - Dinâmica da C@-LMEIAS 
Na Figura 2, o caminho da (re)construção do saber se dará em espiral, partindo do resgate dos conhecimentos prévios, indo dos conceitos gerais para os mais específicos (tracejado em cinza), promovendo a diferenciação progressiva dos conteúdos. Contudo, esse caminho não será único. Os alunos poderão traçar os seus próprios trajetos, ao lado de seu professor e de seus colegas (colaboração, tanto presencial como virtual), como é o caso dos percursos percorridos pelo aprendiz da bicicleta e os que se encontram no ônibus (em vermelho e em verde, respectivamente). Haverá uma organização sequencial, porém o discente ao fazer o seu caminho pode pular tópicos e se precisar voltar num conceito, voltará, como ocorreu com os aprendizes no ônibus.

Nesse processo o ambiente de aula se estenderá a todos os contextos onde o aluno estiver buscando o conhecimento (na praça, no ônibus, em casa, na escola, na rua, etc) e a sala será tanto virtual, no Facebook, como presencial, na Instituição de Ensino. Como as pessoas que se encontram no entorno do processo de ensino e de aprendizagem não são objetos estáticos e passivos, como aponta a teoria de Vygotsky, mas companheiros dinâmicos que guiam, regulam, comparam, analisam, selecionam e que registram o desenvolvimento cognitivo, a colaboração entre os aprendizes durante esse processo será incentivada em ambos os ambientes, onde os alunos deverão trabalhar, preferencialmente em grupos, sendo instigados a identificar as relações existentes entre os conceitos, isto é, a reconciliação integradora dos mesmos $(\Delta)$.

Os dispositivos móveis, além de permitirem o contato dos alunos com o ambiente pessoal de aprendizagem, são ferramentas que possibilitarão a pesquisa e a construção de material nesse processo. É através dessas tecnologias que os discentes poderão fotografar os contextos que considerarem relevantes, gravar vídeos e áudios, produzir textos, entre outros.

Portanto a C:-LMEIAS é uma estratégia voltada para a compreensão, baseada na interação dos aprendizes, que procurará facilitar a aprendizagem significativa através da colaboração nas redes sociais em contextos de mobilidade, que empregará o Facebook como uma extensão da sala de aula, já que o mesmo viabiliza o planejamento aberto, flexível, adaptável a dinâmica dos sujeitos, possibilitando uma metodologia adaptada ao contexto e voltadas para a afetividade (Masini, 2010) e que possibilita empregar a mobilidade de forma efetiva (Junior, 2009).

Para o seu sucesso, o professor precisará ser orientador e mediador e o aluno predisposto a ser agente de sua aprendizagem. Por empregar as redes sociais, a metodologia necessitará ser organizada e ao mesmo tempo adaptável ao contexto. O docente precisará estar disponível online, compartilhar, participar e motivar os aprendizes durante todo o processo.

De uma maneira geral, a estrutura da C(:)LMEIAS ainda consiste nas seguintes etapas: (i) apresentação do conteúdo; (ii) resgate dos conceitos prévios com os estudantes; (iii) distribuição da turma em pequenos grupos; (iv) exploração do assunto pelos aprendizes, a fim de se especificar os conteúdos; (v) proposição pelo professor de situações problemas contextualizados; (vi) solução dos problemas pelos discentes; (vii) ressignificação dos saberes pelo alunos empregando os recursos do Facebook e das tecnologias móveis, através da construção de materiais digitais e desafios; (viii) compartilhamento daquilo que foi elaborado pelos pequenos grupos com o grande grupo (a turma) na "sala" virtual; (ix) discussão dos materiais; (x) resolução dos desafios pelos alunos; (xi) surgimento de novos questionamentos; (xii) recomeço do processo.

Observa-se que na etapa (viii) espera-se que os aprendizes além de curtir e comentar o que os colegas postaram, haja o estabelecimento de um grande diálogo em 
8

rede através de uma comunidade de aprendizagem, descrevendo assim uma situação colaborativa que promove uma aprendizagem significativa.

\section{Considerações Finais}

Os recursos tecnológicos estão cada vez mais diversificados em nosso meio. Vive-se na SI, onde os jovens são nativos digitais. Esses estão familiarizados com os RSI e associá-los à educação é uma das formas de aproximar essa aos contextos desses alunos, a partir de uma metodologia que explore a colaboração entre os pares, que promova a aprendizagem significativa, num contexto de mobilidade.

Diversos são os fatores que a revisão de literatura indicam para fazer essa aproximação, entre eles destaca-se a possibilidade da aprendizagem ocorrer em qualquer lugar, independente do dispositivo e do tempo. A Internet já é acessivel em praticamente todos os ambientes e com a chegada das redes $4 \mathrm{G}$, em futuro próximo, a conectividade será onipresente, permitindo cada vez mais que os processos de aprendizagem com mobilidade sejam estabelecidos. Quando esse processo for associado a uma estratégia adequada, poderá possibilitar uma aprendizagem centrada no aluno, ao longo da vida, integrada, contextualizada, colaborativa, que favorece o pensamento crítico, a autônomia, o aprender a aprender e o aprender pela descoberta.

Perante a isso, a estratégia C(-)LMEIAS, procurará incentivar os alunos a resolverem situações problemas, que visem a descoberta e o desenvolvimento de sua criatividade ao empregarem os recursos associados aos dispositivos móveis para (re)construirem os significados dos conteúdos de forma contextualizada e significativa e elaborarem novos materiais didáticos, trabalhando de forma colaborativa, em grupos. Essa será uma metodologia baseada na interação social, na qual os estudantes trabalharão em conjunto em uma tarefa, trocarão pontos de vista, experiências e opiniões, discutirão e negociarão estratégias, ações e resultados. Todos os materiais deverão ser compartilhados no grande grupo (a turma), através do Facebook, sendo justificados, comentados, curtidos, debatidos, explicados.

Enfim, almeja-se através dessa estratégia didático-pedagógica que um grande diálogo em rede se estabeleça a partir do desenvolvimento dos recursos didáticos, o que caracterizarão as situações de aprendizagem colaborativas, como referenciadas por Dillenbourg (1999), sendo esperado com a mesma que realmente seja alcançado a aprendizagem significativa, como proposto por Santos (2009), uma vez que os conceitos serão trabalhados de maneira que os saberes sejam (re)significados pelos alunos, de forma contextualizada, a partir de situações problemas e desafios, o que promoverá um sentido contextual para os mesmos. Além disso, através da colaboração nos ambientes de aprendizagem espera-se que se desenvolva um sentido emocional por parte dos aprendizes.

Logo, através da C:-)LMEIAS, considera-se que o compartilhamento do conhecimento a partir das redes sociais seja muito maior do que numa aula tradicional, pois um aumento da colaboração entre os discentes é esperado ao empregar-se o Facebook como um PLE. Esse ambiente apoiado pelas tecnologias móveis e por uma metodologia de ensino e de aprendizagem que instigue a aprendizagem colaborativa e significativa dos sujeitos envolvidos, permitirá que os aprendizes definam os seus próprios caminhos de aprendizagem, investigando a própria realidade, através de uma versabilidade de recursos que lhe são familiares, o que poderá, segundo Selbach (2010), ajudar o professor a ensinar e encantar seus alunos, defendendo a ideia de que a Instituição de Ensino, é um espaço privilegiado que deve visar à construção de um saber 
9

que capacite os alunos a pensar e refletir sobre a realidade, assim como a agir e transformá-la.

${ }^{1}$ ou m-learning.

${ }^{2}$ Personal Computer.

${ }^{3}$ Ubiquitous Computing.

${ }_{5}^{4}$ International Data Corporation.

${ }^{5}$ International Telecommunication Union.

${ }^{6}$ Worldwide Interoperability for Microwave Access.

${ }^{7}$ Long Term Evolution.

${ }^{8}$ Personals Learnings Environments (PLEs).

${ }^{9}$ exposto em Moreira (1999).

\section{Referências Bibliográficas}

ALCÂNTARA, P. R. et al. Vivenciando a aprendizagem colaborativa em sala de aula: experiências no ensino superior. Revista Diálogo Educacional, v. 4, n. 12, p.1-20, 2004.

AL-HUSAIN, D.; HAMMO, B. The Role of Mobile Technology and Social Media in Mobile Learning: A Literature Review. In: WORLDCOMP, Las Vegas, Nevada, USA, 2013, p. 1-7.

ALMEIDA, C.; et al. Aprendizagem colaborativa em ambientes baseados na Web. In: ATAS DO V CONGRESO GALEGO-PORTUGUÉS DE PSICOPEDAGOXIA, p. 193-202, 2000.

ASABERE, N. Y. Review of Recommender Systems for Learners in Mobile Social/Collaborative Learning. International Journal of Information and Communication Technology Research, v. 2, n.5, p. 429-435, 2012.

BARCELOS, G. T.; PASSERINO, L. M.; BEHAR, P. A. Redes Sociais na Internet: Ambiente Pessoal de Aprendizagem na Formação de Professores Iniciantes de Matemática. RENOTE, v.9, n.1, 2011.

COBCROFT, R.; et al. Mobile learning in review: Opportunities and challenges for learners, teachers, and institutions. In: ONLINE LEARNING AND TEACNHING (OLT) CONFERENCE, Queensland University of Technology, Brisbane, 2006, p. 2130.

COLL, C.; MONEREO, C. Educação e aprendizagem no século XXI: novas ferramentas. In: COLL C.; MONEREO C. Piscologia da educação virtual. São Paulo: Artmed, 2010. p. 15-46.

DILLENBOURG, P. What do you mean by 'collaborative learning'? In: DILLENBOURG, P. (Ed) Collaborative-learning: cognitive and computacional approaches. Oxford: Elsevier, 1999. p. 1-19.

ESPADA, A. B. Redes Sociais e o Impacto na Eucação. Janus- III SEDIES 2012, v. 9, n. 15, p. 45 -56, 2012.

FRÍAS, E. R.; MONTAÑO, J. L. A. Exploring the Use of Social Network Sites on Accounting Education: A Social Constructivist Approach. VI Jornada de Docencia en Contabilidad, La Formación en Contabilidade en los Grados y Masters, España, 2012.

FORMAN, G. H.; ZAHORJAN, J. The Challenges of Mobile Computing. IEEE, 1994. HART, J., HANNAN, M. The future of mobile technology and mobile wireless computing. Emerald, v. 21, n. 5, p.201-204, 2004. 
JUNIOR, P. G. G. Aprendizagem com mobilidade (m-learning) na perspectiva dialógica: reflexões e possibilidades para práticas pedagógicas. RENOTE, v.7, n.3, 2009.

KENSKI, V. M. Tecnologias e ensino presencial e a distância. Campinas: Papirus, 2010.

LAM, J.; YAU, J.; CHEUNG, S.K.S. A Review of Mobile Learning in the Mobile Age. Third International Conference, ICHL 2010, p. 306-315, 2010.

LAOURIS, Y. We need an educationally relevant definition of mobile learning. Proceedings of mLearn, 2005.

MASCOLO, C. The power of mobile computing in a social era. IEEE Computer Society, 2010.

MASINI, E. F. S. Aprendizagem por compreensão e reflexão. In: MASINI, E. F. S.; PEÑA, M. D. J. (org.). Aprendendo significativamente: uma construção colaborativa em ambientes de ensino presencial e virtual. São Paulo: Vetor, 2010. p.14-36.

MORAN, J. M. A educação superior a distância no Brasil. In: SOARES, M. S. A. (org). A Educação Superior no Brasil. Brasília, DF: Coordenação de Aperfeiçoamento de Pessoal de Nível Superior, 2002. p. 251-274.

MOREIRA, M. A. Teorias de Aprendizagem. São Paulo: EPU, 1999.

PEÑA, M. D. J. (org.). Aprendendo significativamente: uma construção colaborativa em ambientes de ensino presencial e virtual. São Paulo: Vetor, p.14-36, 2010.

OLIVEIRA, C. A.; PIMENTEL, F. S. C.; MERCADO, L. P. L. Estágio Supervisionado em Matemática e Redes Sociais: O Facebook no Ensino-Aprendizagem. Revista Educação a Distância e Práticas Educativas Comunicacionais e Interculturais EDaPECI, v. 7, n. 7, ano III, 2011.

PAIXÃO, A. F.; et al. Redes Sociais e Educação: o Facebook Enquanto um Espaço com Potencialidades para o Ensino Superior de Matemática? In: TICEDUCA, Lisboa, 2012, p. 2423 - 2435.

PETROVIC, N.; et al. Possibile Educational Use of Facebook in Higher Environmental Education. Proceedings ICICTE 2012, p. 355-362, 2012.

SANTOS, J. C. F. dos. Aprendizagem significativa: modalidades de aprendizagens e o papel do professor. Porto Alegre: Mediação, 2009.

SATYANARAYANAN, M. Fundamental challenges in mobile computing. ACM, 1996.

SRIVASTAVA, H.; SRIVASTAVA S. Future Wireless Technology - 4G Network. Science Horizon, v. 2, n. 7, 2012.

SELBACH, S. (org). Matemática e didática. Petrópolis, RJ: Vozes, 2010.

TRAXLER, J. Defining, Discussing, and Evaluating Mobile Learning: The moving finger writes and having writ... IRRODL, v. 8, n. 2, p.1-12, 2007.

TRIFONOVA, A. Mobile Learning - Review of the Literature. Technical Report DIT03-009, March 2003.

WINTERS, N. What is mobile learning? In: SHARPLES, Mike. Big Issues in Mobile Learning. University of Nottinham, 2006. p. 5-9.

WU, W. H.; et al. Review of trends from mobile learning studies: A meta-analysis. Computers \& Education, v. 59, n. 2, p. 817-827, 2012.

YU, Y.; ZHU, Y. Review of Empirical Researches on Social Network Sites. IMETI, 2011. 